\title{
Does the mitral valve prosthesis adversely affect the hemodynamic performance of the aortic valve prosthesis in patients with double valve replacement?
}

\author{
Konstantinos Spiliotopoulos, MD, ${ }^{\mathrm{a}}$ Susan Armstrong, MSc, ${ }^{\mathrm{a}}$ Manjula Maganti, MSc, ${ }^{\mathrm{a}}$ and \\ Tirone E. David, MD ${ }^{\text {a }}$
}

\begin{abstract}
Objective: This study was undertaken to examine the possible adverse effect of the mitral valve prosthesis on the hemodynamic performance of the aortic valve prosthesis in patients who have undergone double valve replacement.
\end{abstract}

\begin{abstract}
Methods: Patients who underwent double valve replacement were matched for age, body surface area, left ventricular function, and size and type of aortic valve prosthesis with patients who underwent isolated aortic valve replacement. Two types of prosthetic valves were examined: the St Jude Medical mechanical valve (St Jude Medical, St Paul, Minn) and the Hancock II bioprosthesis (Medtronic Inc, Minneapolis, Minn). Five patients for each size and type of aortic valve prosthesis in the double valve replacement group were matched at 1:2 with patients in the isolated aortic valve replacement group. Only valve sizes 21 to 27 were matched. Hemodynamic assessment of the aortic valve prosthesis was performed by transthoracic echocardiogram before hospital discharge.
\end{abstract}

Results: Matched patients had similar clinical profiles. There were no differences in the systolic gradients, effective aortic valve areas, or flow velocity across the aortic valve prostheses after isolated aortic valve replacement or double valve replacement.

Conclusions: Early after surgery, the hemodynamic performance of aortic valve prostheses was not affected by the presence of mitral valve prostheses in patients who underwent combined aortic and mitral valve replacement. (J Thorac Cardiovasc Surg 2012;143:S74-7)

Replacement of the aortic and mitral valves is a complex operation and requires greater anatomic knowledge of the left ventricular inflow tract and let ventricular outflow tract (LVOT) because of the anatomic continuity between the aortic and mitral valves. This is particularly true when the mitral valve is replaced by bioprosthetic valves with stents that may protrude into the LVOT if incorrectly oriented during implantation. A study ${ }^{1}$ published in 1980 on the hemodynamic features of 7 patients who underwent combined aortic valve replacement (AVR) and mitral valve replacement (MVR), that is, double valve replacement (DVR), with pericardial bioprostheses suggested that the effective orifice areas were similar to those of historical controls of patients who underwent isolated AVR.

From the Division of Cardiovascular Surgery of the Peter Munk Cardiac Centre at Toronto General Hospital, ${ }^{\text {a }}$ Toronto, Ontario, Canada.

Disclosures: Konstatinos Spiliotopoulos, Susan Armstrong, Manjula Maganti, and Tirone E. David have nothing to disclose with regard to commercial support.

Presented at The American Association for Thoracic Surgery Mitral Conclave, New York, New York, May 5-6, 2011.

Received for publication May 1, 2011; revisions received Sept 21, 2011; accepted for publication Oct 20, 2011; available ahead of print Nov 21, 2011.

Address for reprints: Tirone E. David, MD, 200 Elizabeth St 4N457, Toronto, Ontario, Canada M5G 2C4 (E-mail: tirone.david@uhn.on.ca).

$0022-5223 / \$ 36.00$

Copyright (c) 2012 by The American Association for Thoracic Surgery

doi:10.1016/j.jtcvs.2011.10.030
We hypothesized that the presence of a mitral valve prosthesis in patients who have undergone DVR adversely affects the hemodynamic performance of the aortic valve prosthesis because it interferes with the movements of the intervalvular fibrous body during systole. In addition, certain mitral valve prostheses protrude into the LVOT even when correctly implanted. We could not find any information in the medical literature regarding this issue. Thus, we designed a retrospective study whereby we compared the hemodynamic performance of aortic valve prostheses in patients who underwent DVR with that of similar aortic valve prostheses in patients who underwent isolated AVR.

\section{PATIENTS AND METHODS}

Patients' clinical and operative data and in-hospital outcomes were collected prospectively and entered into a computerized database. Our institutional review ethics board approved this retrospective study, and patients' consents were waived. We reviewed the records of all patients who underwent DVR between January 1, 1990, and August 31, 2008, with the same type of prosthetic valve in the mitral and aortic positions. Only patients who left the hospital alive without early reoperation were included in the study. Two types of prosthetic valves were examined: the St Jude Medical mechanical valve (St Jude Medical, St Paul, Minn) and the Hancock II bioprosthesis (Medtronic Inc, Minneapolis, Minn). Patients with a preoperative or postoperative left ventricular ejection fraction less than $40 \%$ and patients who had cardiac procedures other than DVR with or without coronary artery bypass were excluded. We randomly selected 5 patients who underwent DVR for each type and size of prosthetic aortic valve, and 


\section{Abbreviations and Acronyms}

AVR $=$ aortic valve replacement

$\mathrm{DVR}=$ double valve replacement

$\mathrm{MVR}=$ mitral valve replacement

manually matched them at 1:2 with patients who underwent isolated AVR for valve size and type, age ( \pm 10 years), body surface area $( \pm 10 \%)$, and left ventricular ejection fraction $(40 \%-54 \%$ and $\geq 55 \%$ only; those with an ejection fraction $<40 \%$ were excluded). Thus, we included 20 patients who underwent DVR and 40 patients who underwent isolated AVR for each type of valve. After these patients were matched, we retrieved their echocardiograms taken before hospital discharge. The following hemodynamic data on the aortic valve prostheses were examined: aortic valve area, peak systolic gradient, mean gradient, flow velocity across the aortic valve, and aortic regurgitation. We collected the hemodynamic data on the mitral valve prosthesis in patients who underwent DVR and examined and compared the preoperative risk profile of the 2 cohorts of patients (AVR and DVR).

\section{Statistical Analysis and Presentation}

All statistical analyses were conducted with SAS 9.1 software (SAS Institute Inc, Cary, NC). ${ }^{2}$ Categoric variables were analyzed using the chi-square or Fisher exact test and are expressed as percentages. Continuous variables with normal distribution were analyzed by the Student $t$ test, and the Wilcoxon rank-sum test was used for continuous variables with skewed distribution. Data from continuous variables are reported as mean \pm standard deviation or median where appropriate.

\section{RESULTS}

The preoperative risk profile of the 2 patient cohorts (AVR and DVR) was generally similar, with the exception of a higher prevalence of symptoms of congestive heart failure and a higher incidence of atrial fibrillation in the DVR group compared with the AVR group (Table 1).

The hemodynamic data of the aortic prosthesis in the DVR and AVR groups are shown in Table 2. The hemodynamic performance of the aortic valve prosthesis was similar for every size whether the mitral valve had been replaced or not, negating our hypothesis that the mitral valve prosthesis has a detrimental effect on the hemodynamics of the aortic valve prosthesis in patients who underwent DVR. We could not identify any other variable, such as aortic insufficiency or the hemodynamic performance of the mitral valve prosthesis, to explain these findings.

\section{DISCUSSION}

DVR is a complex operation associated with a higher operative mortality than isolated AVR or MVR. ${ }^{3}$ In a recent report on the perioperative outcomes of heart valve surgery in 623.039 patients from the database of the Society of Thoracic Surgeons, DVR represented only $6.3 \%$ of all valve operations and was associated with an overall operative mortality of $10.7 \%$, whereas the operative mortality was $4.9 \%$ for AVR and $6.3 \%$ for MVR. ${ }^{3}$ Numerous patient variables affect operative mortality in DVR, but technical difficulties in replacing both valves also play an important role. The senior author of this study has performed reoperations in several patients who underwent DVR at other institutions, and severe LVOT obstruction developed because of incorrect orientation of the mitral valve bioprosthesis. There are no data on how often this technical error occurs, but experienced surgeons know that MVR with high-profile valves can be a problem, particularly in female patients with mitral stenosis and a small left ventricular cavity. In addition, bioprosthetic mitral valve stents have also been blamed for some cases of left ventricular wall rupture after MVR. $^{4}$

Implantation of a prosthetic valve in the mitral position deforms the intervalvular fibrous body and makes it rigid. In addition, all prosthetic mitral valves protrude to some degree into the LVOT. During DVR, the MVR is performed first, and visual inspection of the LVOT before implanting the aortic valve prosthesis invariably shows some degree of protrusion of the mitral valve prosthesis into the LVOT, particularly when the intervalvular fibrous body is short. The mitral valve prosthesis narrows the diameter of the LVOT and prevents the intervalvular fibrous body from moving away from the interventricular septum during systole. If the diameter of the aortic annulus and outflow tract is sized before and after implantation of the mitral valve prosthesis during DVR, there is often a reduction in aortic valve size selection, particularly in patients with rheumatic heart disease and a small ventricular cavity.

For these reasons, we have postulated that the anatomic abnormality caused by the presence of a prosthetic mitral valve adversely affects the hemodynamic function of the aortic valve prosthesis in patients who have undergone DVR. To verify this hypothesis, the present study was conducted, whereby we compared the hemodynamics of the aortic valve prosthesis in patients who underwent DVR with those of patients who underwent isolated AVR with the same size and type of prosthetic valve. The results of this study failed to confirm our hypothesis, because the hemodynamics of the prosthetic aortic valves seem to be similar whether they are implanted in isolation or in combination with mitral valve prostheses.

\section{Study Limitations}

As scientifically sound as we believed our hypothesis to be, we could not demonstrate its validity in the cohort of patients studied. Therefore, it is possible that our hypothesis was erroneous, and that the alterations in the LVOT caused by fixation of the intervalvular fibrous body with rigid mitral valve prostheses and the variable degree of protrusion of the prostheses into the LVOT are irrelevant to the hemodynamic function of prosthetic aortic valves in patients with DVR. However, it is also possible we could not prove our hypothesis because prosthetic aortic valves 
TABLE 1. Cardiac profile and valve pathophysiology of patients

\begin{tabular}{|c|c|c|c|c|c|c|c|c|c|c|c|c|}
\hline \multirow{2}{*}{$\frac{\text { Aortic valve size }}{\text { Variable }}$} & \multicolumn{3}{|c|}{21} & \multicolumn{3}{|c|}{22} & \multicolumn{3}{|c|}{25} & \multicolumn{3}{|c|}{27} \\
\hline & AVR & DVR & $P$ & AVR & DVR & $\boldsymbol{P}$ & AVR & DVR & $\boldsymbol{P}$ & AVR & DVR & $P$ \\
\hline \multicolumn{13}{|l|}{$\begin{array}{l}\text { St Jude Medical (St Jude Medical, } \\
\text { St Paul, Minn) }\end{array}$} \\
\hline \multicolumn{13}{|l|}{ Cardiac comorbidity } \\
\hline Hypertension & 60 & 20 & .3 & 70 & 60 & 1 & 40 & 20 & 6 & 50 & 20 & .6 \\
\hline Dyslipidemia & 50 & 40 & 1 & 70 & 20 & .1 & 40 & 40 & 1 & 30 & 20 & 1 \\
\hline Congestive heart failure & 20 & 100 & .007 & 10 & 100 & .002 & 20 & 80 & .09 & 40 & 60 & .6 \\
\hline Atrial fibrillation/flutter & 20 & 80 & .09 & 0 & 60 & .02 & 0 & 40 & .1 & 0 & 60 & .02 \\
\hline NYHA class IV & 20 & 0 & .5 & 10 & 0 & 1 & 10 & 20 & 1 & 30 & 20 & 1 \\
\hline \multicolumn{13}{|l|}{ Valve pathophysiology } \\
\hline Aortic stenosis & 90 & 20 & .02 & 80 & 40 & .3 & 70 & 20 & .17 & 30 & 0 & .07 \\
\hline Insufficiency & 0 & 40 & & 0 & 20 & & 10 & 40 & & 70 & 60 & \\
\hline Mixed & 10 & 40 & & 20 & 40 & & 20 & 40 & & 0 & 40 & \\
\hline \multicolumn{13}{|l|}{ AV pathology } \\
\hline Rheumatic & 0 & 100 & .001 & 0 & 100 & .001 & 0 & 80 & .0007 & 10 & 0 & .6 \\
\hline Calcified & 50 & 0 & & 50 & 0 & & 20 & 0 & & 0 & 20 & \\
\hline Bicuspid & 50 & 0 & & 50 & 0 & & 60 & 0 & & 30 & 20 & \\
\hline $\begin{array}{l}\text { Prosthetic dysfunction/ } \\
\text { congenital/other }\end{array}$ & & & & & & & 20 & 20 & & 60 & 60 & \\
\hline \multicolumn{13}{|l|}{ MV disease } \\
\hline Stenosis & N/A & 80 & N/A & N/A & 100 & N/A & N/A & 40 & N/A & N/A & 0 & N/A \\
\hline Regurgitation & N/A & 20 & & N/A & 0 & N/A & N/A & 20 & & N/A & 100 & \\
\hline Mixed & N/A & 0 & & N/A & 0 & N/A & N/A & 40 & & & & \\
\hline \multicolumn{13}{|l|}{ MV pathology } \\
\hline Rheumatic & N/A & 80 & N/A & N/A & 100 & N/A & N/A & 80 & N/A & N/A & 20 & \\
\hline Ischemic/myxoma & N/A & 20 & & N/A & 0 & N/A & N/A & 20 & & N/A & 80 & N/A \\
\hline \multicolumn{13}{|l|}{$\begin{array}{l}\text { Hancock II bioprosthesis (Medtronic } \\
\text { Inc, Minneapolis, Minn) }\end{array}$} \\
\hline \multicolumn{13}{|l|}{ Cardiac comorbidity } \\
\hline Hypertension & 70 & 100 & .5 & 60 & 40 & .6 & 60 & 40 & 6 & 70 & 80 & 1 \\
\hline Dyslipidemia & 80 & 40 & .2 & 70 & 20 & .1 & 70 & 20 & .1 & 80 & 60 & .6 \\
\hline Congestive heart failure & 40 & 100 & .04 & 40 & 100 & .04 & 20 & 100 & .007 & 10 & 60 & .08 \\
\hline Atrial fibrillation/flutter & 0 & 60 & .02 & 20 & 40 & 6 & 10 & 40 & .2 & 10 & 40 & .2 \\
\hline NYHA class IV & 10 & 20 & 1 & 40 & 20 & .6 & 20 & 40 & 6 & 30 & 20 & 1 \\
\hline \multicolumn{13}{|l|}{ Valve pathophysiology } \\
\hline Aortic stenosis & 90 & 40 & .08 & 60 & 40 & .5 & 100 & 20 & .004 & 60 & 40 & 1 \\
\hline Insufficiency & 10 & 20 & & 0 & 20 & & 0 & 60 & & 30 & 40 & \\
\hline Mixed & 0 & 40 & & 40 & 40 & & 0 & 20 & & 10 & 20 & \\
\hline \multicolumn{13}{|l|}{ AV pathology } \\
\hline Rheumatic & 0 & 80 & .001 & 10 & 80 & .1 & 0 & 20 & .02 & 0 & 40 & .1 \\
\hline Calcified & 70 & 0 & & 50 & 20 & & 60 & 40 & & 60 & 20 & \\
\hline Bicuspid & 20 & 20 & & 20 & 0 & & 40 & 0 & & 20 & 20 & \\
\hline $\begin{array}{l}\text { Prosthetic dysfunction/ } \\
\text { congenital/other }\end{array}$ & 10 & 0 & & 20 & 0 & & 0 & 40 & & 20 & 20 & \\
\hline \multicolumn{13}{|l|}{ MV disease } \\
\hline Stenosis & N/A & 40 & N/A & N/A & 80 & N/A & N/A & 0 & N/A & N/A & 20 & N/A \\
\hline Regurgitation & N/A & 20 & & N/A & 20 & N/A & N/A & 80 & & N/A & 40 & \\
\hline Mixed & N/A & 40 & & N/A & 0 & N/A & N/A & 20 & & & 40 & \\
\hline \multicolumn{13}{|l|}{ MV pathology } \\
\hline Rheumatic & N/A & 100 & N/A & N/A & 100 & N/A & N/A & 20 & N/A & N/A & 60 & N/A \\
\hline $\begin{array}{l}\text { Other (ischemic, myxomatous, } \\
\text { prosthetic dysfunction) }\end{array}$ & N/A & 0 & & N/A & 0 & N/A & N/A & 80 & & N/A & 40 & \\
\hline
\end{tabular}


TABLE 2. Demographics and aortic prosthesis hemodynamics of patients

\begin{tabular}{|c|c|c|c|c|c|c|c|c|c|c|c|c|}
\hline \multirow{2}{*}{$\begin{array}{c}\text { Aortic valve size } \\
\text { Variable }\end{array}$} & \multicolumn{3}{|c|}{21} & \multicolumn{3}{|c|}{23} & \multicolumn{3}{|c|}{25} & \multicolumn{3}{|c|}{27} \\
\hline & AVR & DVR & $P$ & AVR & DVR & $\boldsymbol{P}$ & AVR & DVR & $P$ & AVR & DVR & $P$ \\
\hline \multicolumn{13}{|l|}{ St Jude Medical } \\
\hline Age (y) & $63 \pm 7$ & $63 \pm 6$ & 1 & $61 \pm 5$ & $61 \pm 5$ & 1 & $57 \pm 8$ & $58 \pm 10$ & .8 & $42 \pm 11$ & $42 \pm 12$ & .9 \\
\hline BSA & $1.66 \pm 0.12$ & $1.62 \pm 0.12$ & .6 & $1.99 \pm 0.14$ & $1.99 \pm 0.2$ & 1 & $1.98 \pm 0.16$ & $2.03 \pm 0.18$ & .6 & $2.2 \pm 0.3$ & $2.2 \pm 0.1$ & .9 \\
\hline Female $(\%)$ & 70 & 80 & 1 & 10 & 60 & .08 & 10 & 0 & 1 & 0 & 0 & N/A \\
\hline AVA $\mathrm{cm}^{2}$ & $1.39 \pm 0.18$ & $1.51 \pm 0.26$ & .3 & $1.33 \pm 0.22$ & $1.3 \pm 0.2$ & .8 & $1.50 \pm 0.27$ & $1.29 \pm 0.11$ & .1 & $1.50 \pm 0.48$ & $1.48 \pm 0.3$ & .9 \\
\hline PG mm Hg & $28.5 \pm 7.6$ & $24.7 \pm 9.2$ & .5 & $28.3 \pm 8.2$ & $22.8 \pm 6.8$ & .2 & $26.7 \pm 13.9$ & $22.6 \pm 6.5$ & .8 & $24.2 \pm 6.1$ & $26.2 \pm 5.7$ & .4 \\
\hline MG mm Hg & $13.6 \pm 3.7$ & $11.6 \pm 5.1$ & .4 & $14.3 \pm 5.9$ & $11.2 \pm 4.3$ & .4 & $12.8 \pm 6.9$ & $10.8 \pm 3.5$ & 6 & $12.8 \pm 3.1$ & $13.4 \pm 2.7$ & .7 \\
\hline AvVel m/sec & $2.4 \pm 0.6$ & $2.4 \pm 0.8$ & .8 & $2.6 \pm 0.4$ & $2.4 \pm 0.3$ & .5 & $2.5 \pm 0.7$ & $2.4 \pm 0.5$ & .7 & $2.5 \pm 0.2$ & $2 \pm 0.3$ & .8 \\
\hline \multicolumn{13}{|l|}{ Hancock II bioprosthesis } \\
\hline Age (y) & $75 \pm 4$ & $74 \pm 4$ & .8 & $70 \pm 14$ & $69 \pm 16$ & .9 & $70 \pm 11$ & $70 \pm 12$ & 1 & $71 \pm 9$ & $71 \pm 10$ & 1 \\
\hline BSA & $1.65 \pm 0.17$ & $1.64 \pm 0.17$ & .9 & $1.66 \pm 0.11$ & $1.65 \pm 0.11$ & .9 & $1.94 \pm 0.12$ & $1.87 \pm 0.12$ & .3 & $1.93 \pm 0.14$ & $1.95 \pm 0.11$ & .8 \\
\hline Female $(\%)$ & 100 & 80 & .3 & 30 & 100 & .03 & 10 & 20 & 1 & 0 & 20 & .3 \\
\hline AVA $\mathrm{cm}^{2}$ & $1.2 \pm 0.3$ & $1.14 \pm 0.2$ & .6 & $1.3 \pm 0.24$ & $1.24 \pm 0.1$ & .7 & $1.39 \pm 0.22$ & $1.2 \pm 0.23$ & .2 & $1.4 \pm 0.24$ & $1.4 \pm 0.35$ & .9 \\
\hline PG mm Hg & $30.9 \pm 11.8$ & $34.1 \pm 14$ & .9 & $26.8 \pm 8.3$ & $20.4 \pm 4.5$ & .1 & $26 \pm 12$ & $25.4 \pm 6.5$ & .9 & $22.3 \pm 4.5$ & $27.8 \pm 16.5$ & .7 \\
\hline MG mm Hg & $16 \pm 6.6$ & $17.4 \pm 10.5$ & .8 & $13.2 \pm 5.3$ & $11.3 \pm 0.6$ & .9 & $13.7 \pm 6.49$ & $12 \pm 2.45$ & 1 & $12.3 \pm 2.8$ & $12.4 \pm 7.4$ & .5 \\
\hline AvVel m/sec & $2.6 \pm 0.45$ & $2.7 \pm 0.3$ & .7 & $2.5 \pm 0.4$ & $2.2^{*}$ & .5 & $2.5 \pm 0.5$ & $2.5 \pm 0.5$ & 1 & $2.3 \pm 0.2$ & $2.4 \pm 0.1$ & .4 \\
\hline
\end{tabular}

All values are given as mean \pm standard deviation of the mean. $A V R$, Aortic valve replacement; $D V R$, double valve replacement; $N / A$, not applicable; $B S A$, body surface area; $A V A$, aortic valve area; $P G$, peak systolic gradient; $M G$, mean systolic gradient; $A v V e l$, flow velocity across aortic valve. *Only 1 patient had flow velocity recorded.

are intrinsically obstructive with effective orifice areas smaller than the actual sizes of the aortic annuluses where they are anchored, and the effect of the mitral valve prosthesis was confounded by the degree of intrinsic obstruction of prosthetic aortic valves.

Other reasons that could explain the outcomes of this comparative study are the limited sample size of patients who have undergone DVR, given the large number of confounding factors that affect blood flow across the LVOT in these patients. In addition, the pathology and the valve lesion were not matched, and most patients who underwent DVR had rheumatic heart valve disease. Finally, the timing of echocardiographic evaluation of the prosthetic aortic valves may play a role on the outcomes. In ideal circumstances, such a study should be conducted after patients have completely recovered from surgery, and the valve hemodynamics should be assessed at rest and during maximum exercise.

\section{CONCLUSIONS}

The presence of mitral valve prosthesis in patients who have undergone DVR has no effect on the early hemodynamic features of the prosthetic aortic valve, as assessed by echocardiography early after surgery.

\section{References}

1. Tandon AP, Whitaker W, Ionescu MI. Multiple valve replacement with pericardial xenograft. Clinical and haemodynamic study. Br Heart J. 1980;44:534-40.

2. SAS Institute, Inc. SAS/STAT User's Guide, Version 8. Cary, NC: SAS Institute, Inc; 1988.

3. Lee R, Li S, Rankin JS, et al. Fifteen-year outcome trends for valve surgery in North America. Ann Thorac Surg. 2011;91:677-84.

4. Nuñez L, Gil-Aguado M, Cerron M, Celemin P. Delayed rupture of the left ventricle after mitral valve replacement with bioprosthesis. Ann Thorac Surg. 1979;27:465-7. 